\title{
Patterns of Range of Motion Restriction in Subjects with Adhesive Capsulitis
}

\author{
Krupa M. Soni ${ }^{1}$, Urmi Bhatt ${ }^{2}$, Vidhya Solanki ${ }^{3}$, Karishma Barot ${ }^{4}$, \\ Priyanka Chaudhari ${ }^{5}$ \\ ${ }^{1}$ Assistant Professor, Nootan College of Physiotherapy, Sankalchand Patel University, Visnagar-384315. \\ Gujarat. \\ ${ }^{2}$ Assistant Professor, C. U. Shah Physiotherapy College, Medical College Campus, Dudhrej Road, \\ Surendranagar-363001, Gujarat. \\ 3, 4, 5 Tutor, Nootan College of Physiotherapy, Sankalchand Patel University, Visnagar-384315. Gujarat.
}

Corresponding Author: Krupa M. Soni

\begin{abstract}
Background: Adhesive capsulitis is one of the most common problems of the arm. It is a painful and disabling condition and the etiology is unclear. Adhesive capsulitis is caused by tightening of the joint capsule and results in stiffness and pain. Adhesive capsulitis occurs in three distinct stages. Freezing stage, Frozen stage \& Thawing stage. Till date, adhesive capsulitis remains to be more of a clinical diagnosis rather than radiological. Cyriax's proposed shoulder capsular pattern was external rotation most limited followed by abduction followed by internal rotation. It remains unclear whether this pattern is exists in all the phases of adhesive capsulitis or not.
\end{abstract}

Aim: To identify pattern of restriction of ROM in subjects with adhesive capsulitis in three phases of adhesive capsulitis.

Methodology: The study included 60 subjects with idiopathic adhesive capsulitis. Passive ROM for shoulder Abduction, internal and external rotation was measured with universal Goniometer.

Results \& Conclusion: Findings suggest that various patterns of restrictions were found in different phase of Adhesive capsulitis.

Key words: Adhesive capsulitis, Shoulder passive ROM, Pattern of restriction

\section{INTRODUCTION}

Adhesive capsulitis is characterised by an insidious and progressive loss of active and passive mobility in glenohumeral joint, presumably due to capsular contracture. ${ }^{[1]}$ Adhesive capsulitis is one of many conditions that present with pain and progressive limitation of active and passive shoulder motion. ${ }^{[2]}$ Adhesive capsulitis has an incidence of $3-5 \%$ in the general population and this disorder is one of the most common musculoskeletal problems seen in orthopaedics. ${ }^{[3,4]}$

Adhesive capsulitis can classify as primary or secondary. Primary (or idiopathic) adhesive capsulitis can occur spontaneously without any specific trauma or inciting event. Secondary adhesive capsulitis is often observed after periarticular fracture dislocation of the glenohumeral joint or other severe articular trauma. ${ }^{[5]}$

Primary adhesive capsulitis is reported to affect $2 \%$ to $5.3 \%$ of the general population. The prevalence of secondary adhesive capsulitis related to diabetes mellitus and thyroid disease is reported to be between $4.3 \%$ and $38 \%$. ${ }^{[6]}$

Adhesive capsulitis was labeled "frozen shoulder" by Codman in 1934 but subsequently termed as "adhesive capsulitis" by Neviaser to better describe the 
pathology. Julius Neviaser through surgical exploration of frozen shoulders found the essential pathology to be thickening and contraction of capsule that became adhered to humeral head. Inferior capsular length is said to be insufficient in subjects with adhesive capsulitis. ${ }^{[2]}$

Adhesive capsulitis has been described as having three sequential phases: a freezing stage, a frozen stage and a thawing or recovery stage. ${ }^{[4]}$

Reeves first divided the clinical presentation of frozen shoulder into three stages in 1975.

Stage I: Freezing stage lasts 2.5 months to 3 months. The patient reports an insidious onset of diffuse shoulder pain, difficulty lying on the affected side and progressive loss of shoulder motion. Patients often identify pain at night. Ache is unrelated to activity and may be worse at rest

Stage II: Frozen stage lasts 4 months to 12 months. The pain gradually subsides and is described as a dull aching type of sensation and occurs at the extreme of their available movement. Loss of motion plateaus with passive motion equal to active motion or gross reduction of glenohumeral movements, with near total obliteration of external rotation (capsular pattern)

Stage III: Thawing stage lasts 12 months to 42 months. This is characterized by the gradual improvement of shoulder motion and the reduction of pain symptoms, mean duration from onset of frozen shoulder to resolution is over 30 months. ${ }^{[7]}$

Pain and limited range of motion can occur in all phases of adhesive capsulitis, which often does not follow a stepwise course. ${ }^{[4]}$

Cyriax initially proposed that tightness in a joint capsule would result in a pattern of proportional motion restriction. Researcher have investigated that External rotation is often the first motion affected on clinical examination, with steady global loss of ROM with disease progression. ${ }^{[5]}$

The shoulder capsular pattern was external rotation (ER) most limited followed by abduction (ABD) followed by internal rotation (IR). The loss of passive ER with the arm at the patient's side is a hallmark of this condition. ${ }^{[8]}$

Cyriax's capsular patterns forms basis for most of the physiotherapy interventions used in treatment of Adhesive capsulitis. Aim \& objective of the study was to observe that the same pattern of capsular restriction in the subjects with Adhesive capsulitis in all the three phase of adhesive capsulitis. To observe weather the percentage of restriction is same in all the three phase of Adhesive capsulitis.

\section{MATERIALS AND METHODOLOGY}

A Cross Sectional Observational study was performed on subjects with Primary Adhesive capsulitis, age between 40 to 70 years ${ }^{[8]}$ with the Convenience sampling. Study was carried out until the desired numbers of subjects were completed, after getting approval from the ethical committee of Nootan College of Physiotherapy, Visnagar. Total 60 subjects with Primary Adhesive capsulitis were included and divided in to the three groups. Group A includes 20 Subjects with Primary Adhesive capsulitis, with the onset of symptoms before 2.5 months to 3 months and have classic features of 1st stage of adhesive capsulitis. Group B includes 20 Subjects with Primary Adhesive capsulitis, with the onset of symptoms before 4 months to 12 months. Group $\mathrm{C}$ includes 20 Subjects with Primary Adhesive capsulitis, with the onset of symptoms before 12 months to 42 months. Subjects who came in OPD of Nootan College of Physiotherapy, Visnagar were included. Subjects with Sign of Radiculopathy ${ }^{[10]}$, any neurological condition affecting shoulder function such as Stroke or Parkinson's disease [11], Subjects taking/ have taken any physiotherapy or surgical treatment ${ }^{[11]}$, Subjects with intrinsic shoulder diseases as biceps tendinitis, rotator cuff tears, history of previous trauma and joint arthritis [11] were excluded. Written consent form was taken with all the Subjects who were willing to participate in the study. All the subjects 
completed a detailed Orthopaedic assessment. Subjects who fulfilled the selection criteria were informed about the study and requested to sign the consent form. Passive ROM of Shoulder joint were performed by only single therapist with universal goniometer. ${ }^{[9]}$ Motion can be performed supine or in the upright position. Unit of measurement was taken in degrees.

\section{Shoulder Abduction}

To measure abduction ROM, the patient is positioned in supine with the arm comfortably by the side. As seen in Graph1 , the examiner passively abducts the shoulder until end range is reached (shoulder must remain in the same plane). ROM is measured by placing the axis of the goniometer on the head of the humerus. The stationary arm is aligned parallel with the midline of the sternum. The movable arm is aligned with the midshaft of the humerus.

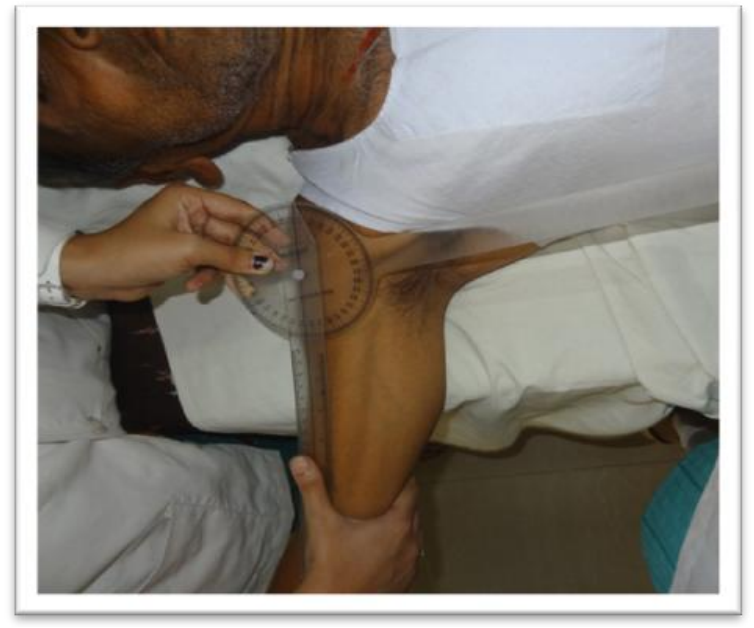

Graph: 1- Passive ROM for Shoulder Abduction.

\section{Glenohumeral Internal Rotation in Abduction}

Internal rotation ROM is measured with the patient positioned in supine, the shoulder abducted to $90^{\circ}$, and the elbow flexed to $90^{\circ}$. If glenohumeral abduction is less than $90^{\circ}$, a $45^{\circ}$ abduction angle can be used. The examiner passively internally rotates the glenohumeral joint until end range is reached, ensuring that there is no scapular compensation. ROM is measured by placing the axis of the goniometer on the olecranon process. The stationary arm is aligned with the vertical position. The movable arm is aligned with the ulnar styloid process.

\section{Glenohumeral External Rotation in Abduction}

External rotation ROM may also be measured with the shoulder abducted to $45^{\circ}$ or to $90^{\circ}$ in the frontal plane (if the patient has the available abduction ROM). The examiner passively externally rotates the glenohumeral joint until end range is reached. ROM is measured by placing the axis of the goniometer on the olecranon process. The stationary arm is aligned with the vertical position. The movable arm is aligned with the ulnar styloid process. ${ }^{[6,10]}$

\section{RESULTS}

PROM of 60 subjects, Mean \& SD of age in Group A 55.2 \pm 8.77 , in Group B Mean age \& SD 54.9 \pm 9.18 \& in Group 3 Mean age \& SD 54.4 \pm 7.06 as shown in table 1, with idiopathic adhesive capsulitis were analyzed by using Microsoft Office Excel version 7 .

Table 1: Demographic details of Mean Age

\begin{tabular}{|c|c|c|c|c|}
\hline \multicolumn{2}{|c|}{ Demographic details } & Group A & Group B & Group C \\
\hline \multirow[t]{2}{*}{ Age } & Mean & 55.2 & 54.9 & 54.4 \\
\hline & SD & \pm 8.77 & \pm 9.18 & \pm 7.06 \\
\hline
\end{tabular}

Table 2: Gender distribution in all groups
\begin{tabular}{|l|c|c|c|}
\hline Groups & Male & Female & \multirow{2}{*}{ Total } \\
\cline { 2 - 3 } & $\mathbf{n}$ & $\mathbf{n}$ & \\
\hline Group A & 8 & 12 & 20 \\
\hline Group B & 9 & 11 & 20 \\
\hline Group C & 8 & 12 & 20 \\
\hline
\end{tabular}

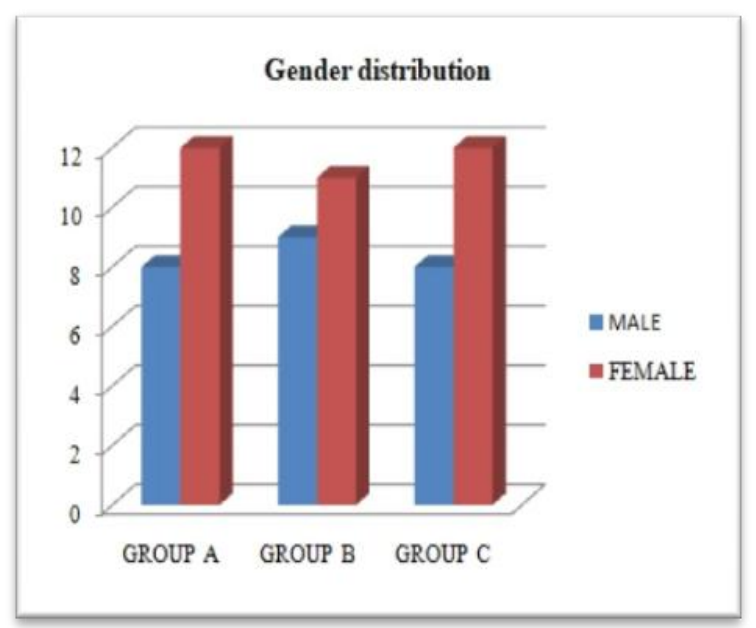

Graph: 2 - Gender distribution among three groups 
Table 2 and Graph 2 shows gender distribution among all three groups, which shows homogeneity among groups.

Table 3 shows the percentage of loss of passive range of motion was calculated from obtained ranges for all 3 ranges. Six different patterns of proportional restriction can be made with the three ranges.

\begin{tabular}{|c|c|c|c|c|c|}
\hline Aable 3: Pattern of Restriction \\
\hline A & B & C & D & E & F \\
\hline Abduction & Abduction & External rotation & External rotation & Internal rotation & Internal rotation \\
\hline External rotation & Internal rotation & Abduction & Internal rotation & External rotation & Abduction \\
\hline Internal rotation & External rotation & Internal rotation & Abduction & Abduction & External rotation \\
\hline
\end{tabular}

For every subject one of the patterns of restriction was identified and then, finally proportion of each pattern was calculated and the results are as shown in table 4 and graph 3 that in stage I (Freezing Stage) the $40 \%$ of subjects had $\mathrm{C}$ pattern of restriction that is External rotation more restricted then abduction followed by internal rotation. The same pattern of restriction was seen in Stage II (Frozen Stage) also. In the stage III (Thawing Stage) pattern of restriction found was A, which was abduction more limited followed by external rotation followed by Internal Rotation.

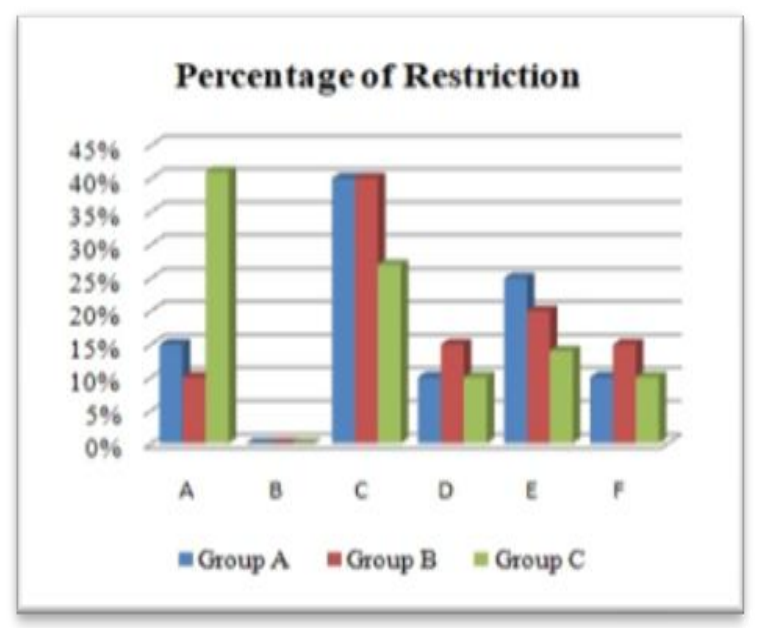

Graph: 3- Pattern of restriction in percentage

Table 4: Proportion of each pattern in percentage

\begin{tabular}{|c|c|c|c|}
\hline \multirow{2}{*}{ Patterns } & $\begin{array}{c}\text { Group A } \\
\text { (Stage I) }\end{array}$ & $\begin{array}{c}\text { Group B } \\
\text { (Stage II) }\end{array}$ & $\begin{array}{c}\text { Group C } \\
\text { (Stage III) }\end{array}$ \\
\hline $\mathrm{A}$ & $15 \%$ & $10 \%$ & $41 \%$ \\
\hline $\mathrm{B}$ & $0 \%$ & $0 \%$ & $0 \%$ \\
\hline $\mathrm{C}$ & $40 \%$ & $40 \%$ & $27 \%$ \\
\hline $\mathrm{D}$ & $10 \%$ & $15 \%$ & $10 \%$ \\
\hline $\mathrm{E}$ & $25 \%$ & $20 \%$ & $14 \%$ \\
\hline $\mathrm{F}$ & $10 \%$ & $15 \%$ & $10 \%$ \\
\hline
\end{tabular}

\section{DISCUSSION}

Current study reveals that more than one pattern of restriction in subjects with adhesive capsulitis in different stages. These findings suggest possibility of involvement of capsule in rather different manner than a uniform manner.

During stage I, there are minimal to no ROM restrictions. Subacromial shoulder impingement is often the suspected clinical diagnosis early in this stage because there are minimal to no ROM restrictions. This finding is correlate with Martin J. Kelley et al 2013, according to the study the arthroscopic examination reveals diffuse synovial reaction without adhesions or contracture. Early loss of external rotation motion with an intact rotator cuff is a hallmark sign of adhesive capsulitis and may be seen in this stage. ${ }^{[6]}$

Stage II can be presents with a gradual loss of motion in all directions due to pain. Martin J Kelly et al (2013), study the subject under anesthesia may reveal the synovitis/angiogenesis lessens but the progressive capsuloligamentous fibrosis results in loss of the axillary fold and ROM restriction pattern may be same as seen in stage I but the amount of restriction is more. [6]

Stage III can be presents with restriction found in abduction more as the pain that begins to resolve, but significant stiffness persists from 15 to 24 months after onsets of symptoms. The findings by martin J Kelly at al arthroscopy reveal capsuloligamentous complex fibrosis and receding synovial involvement. ${ }^{[6]}$ 
The study also suggested as seen in table and Graph 3, involvement of female gender more than male gender.

The study points towards the possibility of tightness in the various components the capsule limiting motion in more than one plane simultaneously.

The same study can be performed with larger sample size and with different outcome measures.

\section{CONCLUSION}

The study found 5 different patterns of restriction in subjects with adhesive capsulitis rather than a single pattern of capsular restriction.

\section{Clinical Implication}

Detailed examination of capsular tissue is a must in subjects with adhesive capsulitis in order to specifically target \& stretch affected part of capsule.

\section{Acknowledgement: None}

\section{Conflict of Interest: None}

\section{Source of Funding: None}

\section{Ethical Approval: Approved}

\section{REFERENCES}

1. Vermeulen HM, Obermann WR, Burger $\mathrm{BJ}$, et al. End-range mobilization techniques in adhesive capsulitis of the shoulder joint: a multiple-subject case report. Phys Therapy. 2000; 80; 1204-1213.

2. Julius Neviaser. Adhesive capsulitis of the shoulder: a study of the pathological findings in periarthritis of the shoulder. $\mathrm{J}$ Bone Joint Surg Am. 1945; 27:211-222.

3. Manske R, Prohaska D. Diagnosis and management of adhesive capsulitis. Current
Reviews in Musculoskeletal Medicine. 2008;1(3-4):180-189.

4. Anthony Ewald. Adhesive Capsulitis: A Review. February 15, 2011, Volume 83, Number 4. American Family Physician-417

5. Le HV, Lee SJ, Nazarian A, Rodriguez EK. Adhesive capsulitis of the shoulder:review of pathophysiology and current clinical treatments. Shoulder and elbow. 2017;9(2):75-84.

6. Kelley M, Shaffer M, Kuhn J, Michener L, Seitz A, Uhl T et al. Shoulder Pain and Mobility Deficits: Adhesive Capsulitis. Journal of Orthopaedic \& Sports Physical Therapy. 2013;43(5):A1-A31.

7. Walton J, Russell S. Physiotherapy assessment of shoulder stiffness and how it influences management. Shoulder \& Elbow. 2015;7(3):205-213.

8. https://www.brighamandwomens.org/assets/ BWH/patients-and

families/rehabilitationservices/pdfs/shoulder -adhesive-capsulitis.pdf

9. T'jonck L, Schacke S, Lysens R, Witvrouw E, Delvaux K, Peers K. Intertester and intratester reliability of the standard goniometer and the cybex edi 320 for active and passive shoulder range of motion in normals and patients. 1998 .

10. Norkin, Cynthia C. Measurement of Joint Motion : a Guide to Goniometry. Philadelphia :F.A. Davis, 1995.

11. Kumar D, Bansal D, Sidhu D, Kular D, Sharma D, Jain D et al. An observational study to compare the outcome of local steroid injections and ultrasonic wave therapy in frozen shoulder patients. International Journal of Orthopaedics Sciences. 2018;4(1b):98-101.

How to cite this article: Soni KM, Bhatt U, Solanki V et.al. Patterns of range of motion restriction in subjects with adhesive capsulitis. Int J Health Sci Res. 2021; 11(6):337-341. DOI: https://doi.org/10.52403/ijhsr.20210650 